\title{
Оптимизация составной охлаждающей ветви
}

\author{
(C) И.А. Драбкин \\ АО „Гиредмет“, \\ 119017 Москва, Россия \\ E-mail: igordrabk@gmail.com
}

(Получена 27 декабря 2016 г. Принята к печати 12 января 2017 г.)

Предложен способ расчета составной охлаждающей термоэлектрической ветви в одномерном приближении методом тепловых балансов с эффективными значениями термоэлектрических параметров: электропроводности $\sigma$, коэффициента термоэдс $\alpha$ и теплопроводности $\kappa$. Данный метод позволяет точно учесть температурные зависимости $\sigma, \alpha, \kappa$. Влияние контактных сопротивлений на результирующую эффективность также учитывается точно. Показан способ перехода от расчета ветви к расчету термоэлемента.

DOI: 10.21883/FTP.2017.07.44651.37

Составная охлаждающая термоэлектрическая ветвь может обеспечивать увеличение максимальной разности температур $\Delta T_{\max }$, получаемой на ветви [1]. Методы расчета таких ветвей достаточно сложны [2] и трудоемки. В работе [3] были получены точные выражения для тепловых балансов на концах термоэлектрической ветви при температурно-зависимых термоэлектрических параметрах. Эти выражения содержат эффективные значения этих параметров - электропроводности $\sigma$, коэффициента термоэдс $\alpha$, теплопроводности $\kappa$, которые рассчитываются исходя из температурного поля в ветви. В данной работе дается обобщение метода эффективных тепловых балансов, позволяющее решать любую задачу оптимального построения термоэлектрических охладителей. Предложенная расчетная процедура более проста и позволяет учесть влияние контактных сопротивлений, которыми ранее пренебрегали, хотя они могут существенно ограничивать возможности составной ветви.

Для описания процессов внутри ветви надо решить уравнение [4]

$$
(\nabla, \kappa \nabla T)+(\mathbf{j}, \mathbf{j}) \rho-T(\mathbf{j}, \nabla \alpha)=0,
$$

где $T$ - температура ветви, $\mathbf{j}-$ плотность тока, $\rho$ - удельное сопротивление. Пусть для определенности, что не ограничивает общности, рассматривается $p$-ветвь. Пусть на горячем конце ветви длиной $L$ и сечением $s$ температура $T_{h}$. За счет пропускания тока создается разность температур $\Delta T=T_{h}-T_{c}$, где $T_{c}-$ температура холодного конца ветви. На холодный конец поступает тепловой поток плотностью $Q_{c}$, а с горячего снимается $Q_{h}$. Ограничимся одномерным случаем, и пусть ось $x$ направлена от холодного конца ветви к горячему, тогда плотность тока $j$ и плотность теплового потока $Q$ будут отрицательны, в соответствии с рис. 1 . В уравнениях теплового баланса они положительны, поэтому заменим $j=-j$ и $Q=-Q$. Уравнение (1) в этом случае принимает вид

$$
\frac{d}{d x}\left(\kappa \frac{d T}{d x}\right)+j^{2} \rho+T j \frac{d \alpha}{d x}=0
$$

В [3] было показано, что, решая (2), можно получить уравнения теплового баланса в виде

$$
\left\{\begin{array}{c}
Q_{c}=\alpha_{c . \mathrm{eff}} T_{c} j-j^{2} L \rho_{c . \mathrm{eff}}-\frac{\bar{\kappa} \Delta T}{L} \\
Q_{h}=\alpha_{h . \mathrm{eff}} T_{h} j+j^{2} L \rho_{h . \mathrm{eff}}-\frac{\bar{\kappa} \Delta T}{L}
\end{array},\right.
$$

где $\alpha_{h \text {.eff, }} \rho_{h \text {.eff, }} \alpha_{c \text {.eff, }} \rho_{c \text {.eff }}$ - эффективные значения $\alpha$ и $\rho$ соответственно для горячего и холодного концов ветви, а $\bar{\kappa}$ - среднее по ветви значение теплопроводности. Выражения для самих эффективных значений имеют вид

$$
\begin{aligned}
& \alpha_{h . \mathrm{eff}}=\alpha\left(T_{h}\right)+\frac{1}{T_{h}} \int_{0}^{L} T \frac{d \alpha(T)}{d T} \frac{d T(y)}{d y} \hat{R}(y) d y, \\
& \alpha_{c . \mathrm{eff}}=\alpha\left(T_{c}\right)-\frac{1}{T_{c}} \int_{0}^{L} T \frac{d \alpha(T)}{d T} \frac{d T(y)}{d y} \hat{L}(y) d y, \\
& \rho_{h . \mathrm{eff}}=\frac{1}{L} \int_{0}^{L} \rho(T(y)) \hat{R}(y) d y+\frac{R_{\mathrm{cont}}}{L}, \\
& \rho_{c . \mathrm{eff}}=\frac{1}{L} \int_{0}^{L} \rho(T(y)) \hat{L}(y) d y+\frac{R_{\mathrm{cont}}}{L}, \\
& \hat{R}(y)=\frac{\bar{\kappa}}{L} \int_{y}^{L} \frac{d x}{\kappa(x)}, \\
& \hat{L}(y)=\frac{\bar{\kappa}}{L} \int_{0}^{y} \frac{d x}{\kappa(x)}, \\
& \bar{\kappa}=L / \int_{0}^{L} \frac{d x}{\kappa(T(x))},
\end{aligned}
$$

где символами $T(x)$ или $T(y)$ обозначено распределение температуры вдоль ветви, получаемое из решения уравнения (2), а операторы $\hat{R}(y)$ и $\hat{L}(y)$ равны отношению 


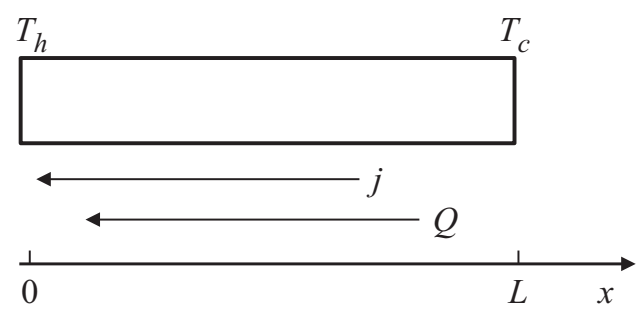

Рис. 1. Схема термоэлектрической ветви.

теплового сопротивления ветви соответственно справа и слева от точки $Y$ к суммарному тепловому сопротивлению ветви, $R_{\text {cont }}-$ контактное сопротивление на концах ветви.

Выражения (4)-(10), в отличие от [1], записаны для удельных сопротивлений и удельной теплопроводности; кроме того, из-за другого направления оси $x$ изменился знак перед вторым членом в (4) и (5). Подчеркнем, что в уравнениях (3) опущен множитель $1 / 2$ перед теплом Джоуля, который представляет собой значения $\rho_{h . \text { eff }}, \rho_{c \text {.eff }}$ для температурно-независимых коэффициентов. Между эффективными величинами $\alpha$ и $\rho$ существуют соотношения [3]

$$
\begin{gathered}
\alpha_{h . \mathrm{eff}} T_{h}-\alpha_{c . \mathrm{eff}} T_{c}=\bar{\alpha} \Delta T, \\
\rho_{h . \mathrm{eff}}+\rho_{c . \mathrm{eff}}=\bar{\rho}+\frac{2 R_{\mathrm{cont}}}{L},
\end{gathered}
$$

где

$$
\begin{gathered}
\bar{\alpha}=\frac{1}{\Delta T} \int_{T_{c}}^{T_{h}} \alpha d T, \\
\bar{\rho}=\frac{1}{L} \int_{0}^{L} \rho(T(x)) d x .
\end{gathered}
$$

В случае зависимости эффективных значений $\alpha, \rho$ и $\bar{\kappa}$ от каких-либо скрытых параметров (концентрации носителей заряда, положения уровня Ферми и т.п.) выражения (4)-(10) можно дифференцировать по этим параметрам и тем самым производить оптимизацию по $Z=\alpha^{2} / \kappa \rho$. Следует подчеркнуть, что, так как имеется по три эффективных значения $\alpha$ и $\rho$, относящихся к холодному и горячему концам ветви, а также их средние значения, для достижения максимальной разности температур необходимо использовать $Z_{c}=\alpha_{c \text {.eff }}^{2} / \bar{\kappa} \rho_{c \text {.eff, }}$ а для максимального холодильного коэффициента $\bar{Z}=\bar{\alpha}^{2} / \overline{\kappa \rho}$.

Рассмотрим теперь составную ветвь из $N$ секций. Нумерация секций и координаты границ ясны из рис. 2.

Обозначим термоэлектрические параметры $i$-секции $(i=1,2, \ldots, N)$ как $\alpha_{i}\left(T_{i}\right), \rho_{i}\left(T_{i}\right), \kappa_{i}\left(T_{i}\right)$, где $T_{i}$ - температура внутри $i$-секции. Соответствующие значения холодных и горячих температур $i$-секции обозначим как $T_{c, i}$ и $T_{h, i}$. Эффективные значения этих термоэлектрических параметров определим по аналогии с выражениями (4)-(10). Для этого надо при интегрировании по координате заменить пределы интегрирования $0 \rightarrow L_{i-1}$, $L \rightarrow L_{i}$, а множитель $1 / L$ перед знаком интеграла необходимо заменить на $1 /\left(L_{i}-L_{i-1}\right)$. Интегрирование по температуре нужно осуществлять в пределах $T_{c, i}$ и $T_{h, i}$, разность температур на $i$-секции равна $\Delta T_{i}=T_{h, i}-T_{c, i}$. $\mathrm{B}$ дальнейшем принадлежность эффективных параметров $i$-секции обозначим индексом, отделенным запятой: $\rho_{h \text {.eff, } i}, \alpha_{c \text {.eff }, i}, \rho_{h \text {.eff }, i}, \rho_{c \text {.eff }, i}$. Среднее значение теплопроводности $i$-секции обозначим $\bar{\kappa}_{i}$. Для операторов $\hat{R}_{i}$ и $\hat{L}_{i}$, относящихся к $i$-секции, справедливы выражения

$$
\begin{aligned}
& \hat{R}_{i}(y)=\frac{\bar{\kappa}_{i}}{L_{i}-L_{i-1}} \int_{y}^{L_{i}} \frac{d x}{\kappa_{i}(x)}, \\
& \hat{L}_{i}(y)=\frac{\bar{\kappa}_{i}}{L_{i}-L_{i-1}} \int_{L_{i-1}}^{y} \frac{d x}{\kappa_{i}(x)},
\end{aligned}
$$

где

$$
\bar{\kappa}_{i}=\left(L_{i}-L_{i-1}\right) / \int_{L_{i-1}}^{L_{i}} \frac{d x}{\kappa_{i}(T(x))},
$$

а тепловое сопротивление $i$-секции, отнесенное к единице сечения, равно

$$
R_{t, i}=\frac{L_{i}-L_{i-1}}{\bar{\kappa}_{i}} .
$$

После того как определены эффективные параметры секций, можно вычислить эффективные параметры ветви, состоящей из $N$ секций: $\alpha_{h N \text {.eff, }} \alpha_{c N \text {.eff, }} \rho_{h N \text {.eff, }} \rho_{c N \text {.eff. }}$ Для эффективных значений $\alpha$ получаем

$$
\begin{aligned}
\alpha_{c N . \mathrm{eff}}= & \alpha_{N}\left(T_{c, N}\right)-\frac{1}{T_{c, N}} \sum_{i=1}^{i=N}\left(\hat{L}_{t a, i} \Delta_{1, i}+\frac{R_{t, i}}{R_{t a}} \Delta_{2, i}\right) \\
& -\frac{1}{T_{c, N}} \sum_{i=2}^{i=N} \hat{L}_{t a, i} \Delta_{3, i}, \\
\alpha_{h N . \mathrm{eff}}= & \alpha_{1}\left(T_{h, 1}\right)+\frac{1}{T_{h}} \sum_{i=1}^{i=N}\left(\hat{R}_{t a, i} \Delta_{1, i}+\frac{R_{t, i}}{R_{t a}} \delta_{2, i}\right) \\
& +\frac{1}{T_{h}} \sum_{i=2}^{i=N} \hat{R}_{t a, i} \Delta_{3, i},
\end{aligned}
$$

где $R_{t a}=\sum_{i=1}^{i=N} R_{t, i}\left(L_{i}-L_{i-1}\right)$ - полное тепловое сопротивление ветви, отнесенное к единице сечения, а

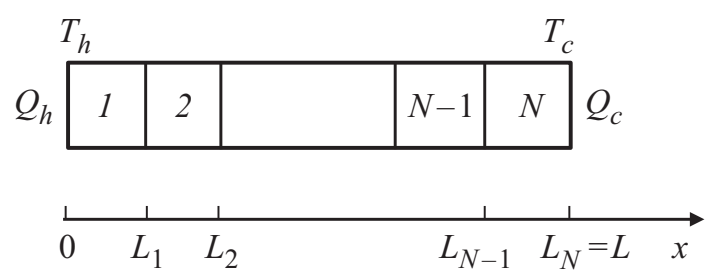

Рис. 2. Схема составной ветви. 
$\hat{R}_{t a, i}=\sum_{k=i+1}^{N} R_{t, i} / R_{t a}$ и $\hat{L}_{t a, i}=\sum_{k=1}^{k=i-1} R_{t, i} / R_{t a}-$ относительное тепловое сопротивление ветви соответственно справа и слева от $i$-секции. Для $\hat{L}_{t a, i}$ и $\hat{R}_{t a, i}$ имеет место тождество

$$
\hat{R}_{t a, i}+\hat{L}_{t a, i}+\frac{R_{t, i}}{R_{t a}}=1
$$

Кроме того, $\hat{R}_{t a, N}=0$ и $\hat{L}_{t a, 1}=0$. Остальные использованные в (18) и (19) обозначения таковы:

$$
\begin{gathered}
\Delta_{1, i}=\alpha_{c, i} T_{c, i}-\alpha_{h, i} T_{h, i}-\bar{\alpha}_{i} \Delta T_{i}, \\
\Delta_{2, i}=\alpha_{h, i} T_{h}^{(i)}-\alpha_{c . \mathrm{eff}, i} T_{c, i}, \\
\Delta_{3, i}=\left(\alpha_{h, i}-\alpha_{c, i-1}\right) T_{c, i}, \\
\delta_{2, i}=\alpha_{h . \mathrm{eff}, i} T_{h, i}-\alpha_{h, i} T_{h, i} .
\end{gathered}
$$

Для эффективных значений сопротивлений составной ветви можно получить

$$
\begin{aligned}
\rho_{c . \mathrm{eff}}= & \sum_{i=1}^{i=N} \hat{L}_{t, i} \bar{\rho}_{i}+\frac{1}{L} \sum_{i=2}^{i=N} \hat{L}_{t, i}\left(R_{\mathrm{cont}, i-1}+R_{\mathrm{cont}, i}\right) \\
& +\frac{1}{R_{t a}} \sum_{i=1}^{i=N} R_{t, i} \rho_{h . \mathrm{eff}, i}, \\
\rho_{h . \mathrm{eff}}= & \sum_{i=1}^{i=N} \hat{R}_{t, i} \bar{\rho}_{i}+\frac{1}{L} \sum_{i=2}^{i=N} \hat{R}\left(R_{\mathrm{cont}, i-1}+R_{\mathrm{cont}, i}\right) \\
& +\frac{1}{R_{t a}} \sum_{i=1}^{i=N} R_{t, i} \rho_{h . \mathrm{eff}, i} .
\end{aligned}
$$

Можно показать, что эффективные значения $\rho$ и $\alpha$ на концах ветви связаны между собой формулами, аналогичными выражениям (11)-(14).

Порядок расчета секционированной ветви таков.

Пусть мы секционируем ветвь для того, чтобы получить максимальное значение $\Delta T_{\max }$. Нам надо найти оптимальный ток и оптимальные длины секций. Вначале мы решаем уравнение (2). К граничным условиям необходимо добавить условия сочленения секций. Будем считать, что на границе секций отсутствует скачок температур, а тепловой поток, выходящий из $i$-секции, поступает в $(i-1)$-секцию, увеличиваясь на джоулево тепло, выделяющееся на контактном сопротивлении. Подробно техника решения такого уравнения дается в работе [2]. При этом вместо уравнения второго порядка решаются два уравнения первого порядка для $d T / d x$ и $d Q / d x$. Следует только иметь в виду, что используемые в данной работе уравнения отличаются знаком $j$. После того как решение найдено в достаточно большом количестве точек, вычисление интегралов, входящих в эффективные значения термоэлектрических параметров, не представляет труда. Значение $\Delta T_{\max }$ находится по стандартным формулам [1]. Затем вычисляются про-

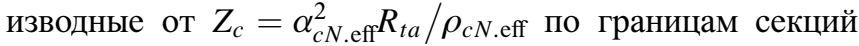
$L_{i}, \quad i=1,2, \ldots, N-1$. Границы секций $L_{i}$ изменяют в соответствии со знаком производной. После этого делается аналогичный расчет для $n$-ветви. Согласование по плотности тока для $p$ - и $n$-ветвей осуществляется изменением сечения ветвей или, если это нецелесообразно, в качестве плотности тока берется их среднее арифметическое. Если же различие в плотностях тока для ветвей различного типа велико и изменять сечение ветвей по каким-то конструктивным соображениям нецелесообразно, то вычисляется $Z_{c}$ термоэлемента и ищется его максимум по границам секций $L_{i}$.

Таким образом находятся основные характеристики составной ветви: оптимальный ток и оптимальные длины секций. Связь эффективных значений на концах ветви со средними по ветви значениями (11)-(14) значительно облегчает отладку программ. Описанная процедура проще, чем используемый в [2] метод максимума Понтрягина, так как там вместо системы двух уравнений для $d T / d x$ и $d Q / d x$ необходимо решать систему из четырех уравнений.

\section{Список литературы}

[1] А.Л. Вайнер, Н.В. Коломоец, Э.М. Лукишкер, В.М. Ржевский. ФТП, 11, 546 (1977).

[2] Л.И. Анатычук, В.А. Семенюк. Оптимальное управление свойствами термоэлектрических материалов и приборов (Черновцы, Прут, 1992).

[3] И.А. Драбкин, 3.М. Дашевский. В сб.: Термоэлектрики и их применения (СПб., 2000) с. 292.

[4] В.Л. Бонч-Бруевич, С.Г. Калашников. Физика полупроводников (М., Наука, 1977) гл. I, с. 20.

Редактор Л.В. Шаронова

\section{Optimization of segmented cooling Leg}

\section{I.A. Drabkin}

JSC „Giredmet”, 119017 Moscow, Russia

Abstract A method of calculating the segmented thermoelectric cooling leg in one-dimensional approximation by means of thermal balance with effective values of thermoelectric parameters conductivity $\sigma$, the Seebeck coefficient $\alpha$ and thermal conductivity $\kappa-$ is presented. This method accurately accounts for the temperature dependences of $\sigma, \alpha, \kappa$. The effect of contact resistances on the efficiency is also taken into account accurately. Application of the method for calculation of performance of thermoelement is also presented. 